\title{
Performance of Turbo Code in CDMA under AWGN Channel
}

\author{
Mohanad Babiker ${ }^{1}$, Othman Omran Khalifa ${ }^{1}$, Aisha Hassan Abdullah Hashim ${ }^{1}$ \\ Momoh J. E. Salami ${ }^{1}$ and Muhammed Zaharadeen Ahmed ${ }^{1}$ \\ Department of Electrical and Computer Engineering, Faculty of Engineering, \\ International Islamic University Malaysia (IIUM), Malaysia \\ Hanode_88@hotmail.com
}

\begin{abstract}
Carrier Division Multiple Access is considered as an efficient technique in wireless communication due to its high possibility to serve an unlimited number of users. CDMA uses the same frequency concurrently with wireless communication. In this paper, the performance and evaluation of turbo code are duly presented followed by the examination of CDMA under AWGN channel was highlighted. The simulation estimated to achieve bit error rate of less than 10-2 frame size.

To this end, the results of the simulation show that the Bit Error Rate is inversely proportional to the size of the frame and directly proportional the number of iteration in the decoder.
\end{abstract}

Keywords: CDMA, BER, Turbo codec

\section{Introduction}

Communication using a wireless medium nowadays becomes a crucial part of our daily living. As a result, degradation of signal, the fast growing number of internet subscribers using wireless equipment's (such as smartphones, laptops etc.,) causes lack of adequate satisfaction by users. In the past ages, several other approaches proposed to curb the issue of degradation of signals. This is mainly to enhance the transmission of electrical signals over a medium for users' satisfaction [1].

However, numerous techniques have been proposed in the most recent century to overcome these issues with a specific objective to provide several users a chance to share the same data transmission concurrently [1].

A technology used in communication systems known as the Code Division Multiple Access (CDMA) is one among the multiple access schemes that permit a wireless equipment to enhance using the same frequency band allocated to multiple users concurrently. It is expected that every communication networks have to ensure a certain level of reliability. For that, channel coding has to be implemented to ascertain that reliability. However, in the situation where the reliability cannot be ascertained at a particular time, an error correcting code technique must be employed to upgrade and engage in retransmission of accurate data being transmitted via the channel [2].

A channel in computer communication systems is a medium of exchange from a transmitter to a receiver over a physical media [2]. Sometimes, a channel can be influenced by a noise or fading in the signal. When this happens, an error message is shown in the course of traffic exchange [2].

However, error correction code is a signal processing technique, which is used for correcting the errors presented as a part of transmitted signal. Different coding techniques are employed in digital and wireless communication. This is to decrease the error in the transmitted and received bits. Therefore, turbo code is the most preferable in the area of coding due to its high efficiency in the application service, which has large transmission 
rate. Turbo code also has high ability to determine a corrupted bit in an accurate manner and hence it is very close to achieving the exact performance of the Shannon capacity [3].

\section{The CDMA2000 Turbo Code}

CDMA is the third generation of wireless communication and cellular system, which institutionalized by the third generation partnership project. The performance of turbo code and turbo decode in CDMA is similar to its performance in the UMTS. The only difference is that the interleaver that is found in both restricts the amount or the size of the frame data, which passes through the devices. The length of the frame is determined in a specific range. Comparing with CDMA, the frame length in UMTS start from 40 bits per frame and reach to up to 5100 bits per frame while in the CDMA the size of the input data has values start from 380 bits per frame and by specific sequence, the frame values reach to 20730 bits per frame [4][5].

Figure 1 below shows a Recursive systematic convolution code (RSC) used by CDMA. The encoder has one input with three-shift register, which produced three outputs (one systematic and two parity) for each input bit. Note that, the code rate of this RSC encoder is $\mathrm{RC}=1 \backslash 3$ (disregarding the tail bits). Note that, the very same path as the parity output of the UMTS encoder creates the principal parity output of the CDMA 2000 encoder. Subsequently, these two encoders just vary by the presence of the second parity output in the CDMA 2000 encoder. The encoder utilized as a part of the parallel linked coding structure $[4,5,6]$.

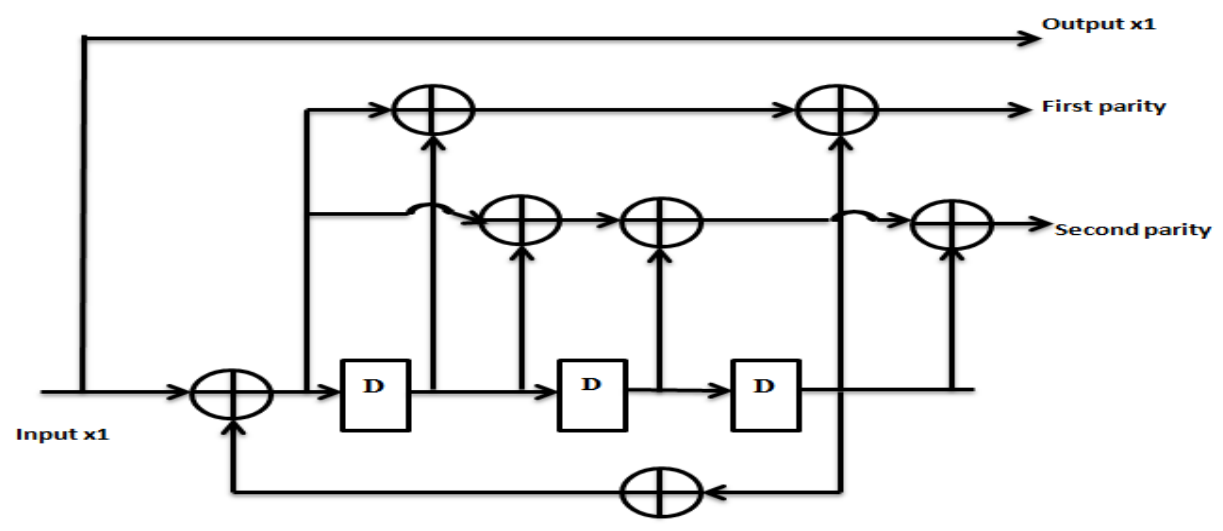

Figure 1. The Simple Encoder used in CDMA2000 (RC=1/30) [1]

\section{Interleaver}

In Turbo coding, the interleaving used to avoid the error in the channel by accepting the input sequence. It also has the ability to spread and rearrange it without repeating or neglecting any individual bit. Therefore, the multiple combinations of the initial sequence are to be carried out using the inverse map in the de-interleaved [7]. There are three ways to read the input sequence, a row column interleaver, helical interleaver and odd even interleaver. Hence, the interleaver that provide bit randomness is preferred most, especially when there is an inputs sequence such as Z1,Z2,Z3,Z4,Z5,Z6,Z7,Z8,Z9 z, and an output which is diagrammatically represented in Figure 2 below. 


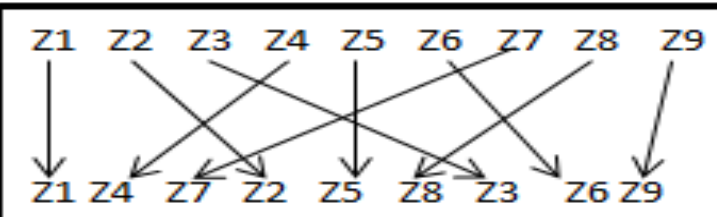

Figure 2. The Re-arranging of Data in the Interleaver

\section{Related Works}

Table 1. Summary of Related Works

\begin{tabular}{|c|c|c|c|}
\hline Author, Year & Technique & Strengths & Limitations \\
\hline $\begin{array}{l}\text { M, Raja Murali } \\
\text { Krishma, } 2014 .\end{array}$ & $\begin{array}{l}\text { Turbo code with } \\
\text { AWGN and fading } \\
\text { channel }\end{array}$ & $\begin{array}{l}\text { Observed improve in } \\
\text { error correcting } \\
\text { coding technique }\end{array}$ & $\begin{array}{l}\text { the trade-off between } \\
\text { the BER and the } \\
\text { number of iterations } \\
\text { need to be made, } \\
\text { output delay is also } \\
\text { longer, The higher } \\
\text { coding rate needs } \\
\text { more bandwidth }\end{array}$ \\
\hline $\begin{array}{l}\text { K. Rasadurai \& N. } \\
\text { kumaratharan, } 2012\end{array}$ & $\begin{array}{l}\text { Turbo MUD SISO } \\
\text { (Soft Input Soft } \\
\text { Output) }\end{array}$ & $\begin{array}{l}\text { Computational } \\
\text { complexity and } \\
\text { greatly improves the } \\
\text { BER performance }\end{array}$ & $\begin{array}{l}\text { Delay can observe } \\
\text { during the simulation }\end{array}$ \\
\hline $\begin{array}{l}\text { Jaswinder singh } \\
\text { 2011[13]. }\end{array}$ & $\begin{array}{l}\text { Fiber-optic CDMA, } \\
\text { Successive } \\
\text { Interference } \\
\text { Cancellation, } \\
\text { Differential Detection }\end{array}$ & $\begin{array}{l}\text { Better than the } \\
\text { conventional TDMA } \\
\text { and FDMA techniques }\end{array}$ & $\begin{array}{l}\text { multiple access } \\
\text { interferences among } \\
\text { the users } \\
\text { simultaneously } \\
\text { accessing the } \\
\text { network }\end{array}$ \\
\hline $\begin{array}{l}\text { R. K. Z. Sahbudin, M. } \\
\text { K. Abdullah (2011). }\end{array}$ & $\begin{array}{l}\text { Khazani-Syed code } \\
\text { with Complementary } \\
\text { subtraction detection }\end{array}$ & $\begin{array}{l}\text { The proposed system, } \\
\text { improve the overall } \\
\text { system performance. }\end{array}$ & $\begin{array}{l}\text { the optical modulation } \\
\text { index per subcarrier } \\
\text { decreases linearly } \\
\text { with the number of } \\
\text { channels }\end{array}$ \\
\hline $\begin{array}{l}\text { Ali Reza Enayati, } \\
\text { Paezi Azim, Yaghoob } \\
2010 \text { [12]. }\end{array}$ & $\begin{array}{l}\text { Multiple user } \\
\text { detections (MUD), } \\
\text { turbo coding and smart } \\
\text { antennas }\end{array}$ & $\begin{array}{l}\text { better performance } \\
\text { compared to linear } \\
\text { MUD algorithms }\end{array}$ & $\begin{array}{l}\text { computational } \\
\text { complexity and delay } \\
\text { grows exponentially } \\
\text { with the number of } \\
\text { users and constellation } \\
\text { size }\end{array}$ \\
\hline $\begin{array}{l}\text { C.M. Negi et al } \\
\text { 2011[11]. }\end{array}$ & $\begin{array}{l}\text { Different Detection } \\
\text { Techniques and } \\
\text { Coding Schemes }\end{array}$ & $\begin{array}{l}\text { MDW coding and } \\
\text { complementary } \\
\text { subtraction shows best } \\
\text { results }\end{array}$ & $\begin{array}{l}\text { The performance of } \\
\text { the OCDMA system } \\
\text { decreases as the bit } \\
\text { rate increases. }\end{array}$ \\
\hline
\end{tabular}

\section{Methodology}

In this paper, turbo code is fully employed and then will be implemented in simulation using MATLAB 2014a. Our methodology parameters are parallel recursive systematic convolution code with code rate $(1,3$, and 3$)$ and modulations 16 QPSK. Eb/no that is organized from (- $1 \mathrm{db}$ to $-6 \mathrm{db})$. A log likelihood ratio algorithm will then be used in the 
demodulation. The length of frame size arranged from 380 to 6140 bits. By using these parameters, the turbo code will test with different frame length to observe the change in the BER, then the number of iteration will change and the BER will be evaluated then be evaluated using graphs. The figure below presents the algorithm in a flowchart view.

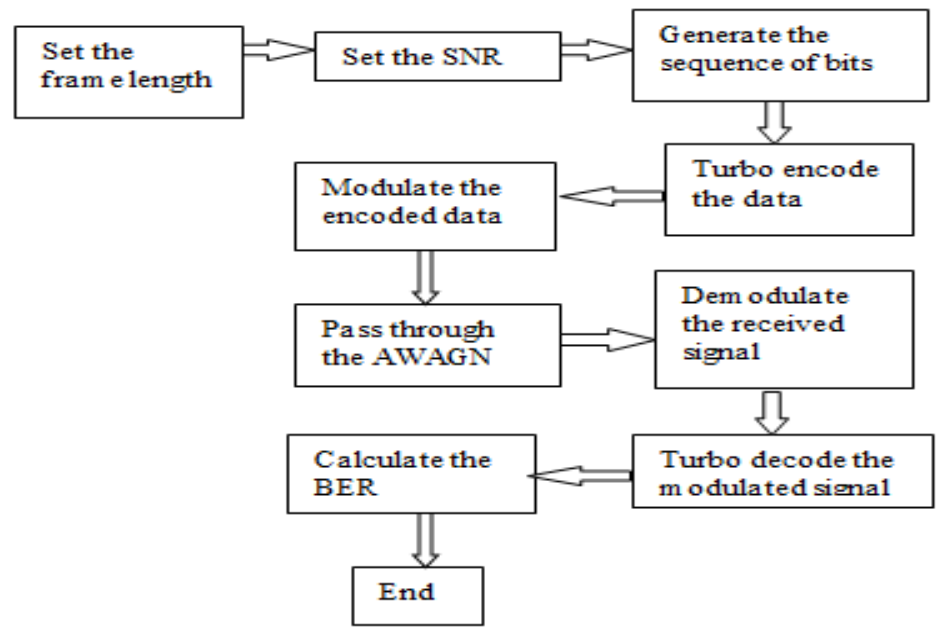

Figure 3. Proposed Simulation Process

\section{Result and Discussion}

The results of Turbo code performance under AWGN channel was achieved used MATLAB software simulations as it shown in tables. The simulation uses the Turbo recursive systematic Convolutional encoder with a constraint length 4 and generator polynomial $[13,15]$ as well as a shift registers memory of 3 . The frame size was arranged from 380 to 6140 bits/frame. We can observe that the Turbo code's performance becomes better under the AWGN channel by observing the simulations.

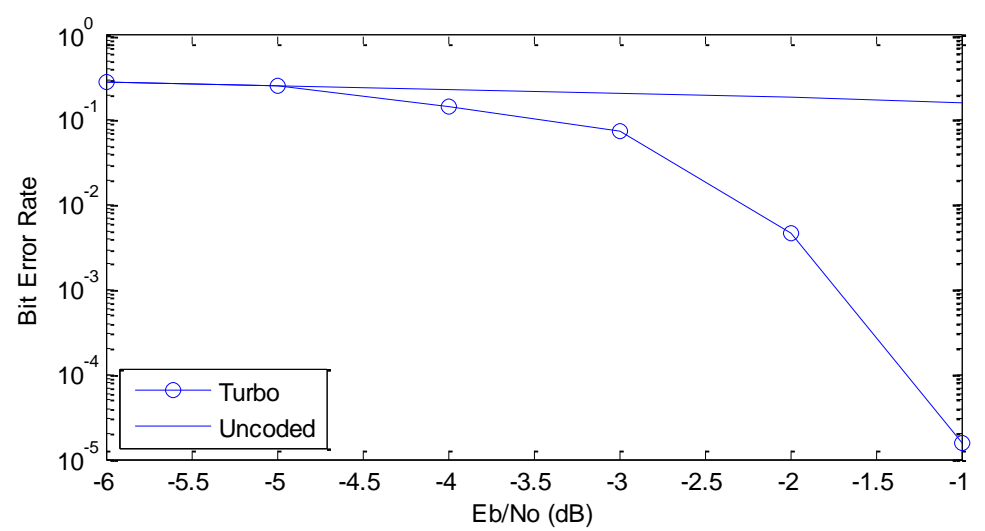

Figure 4. BER with Frame Size 380 Bits 


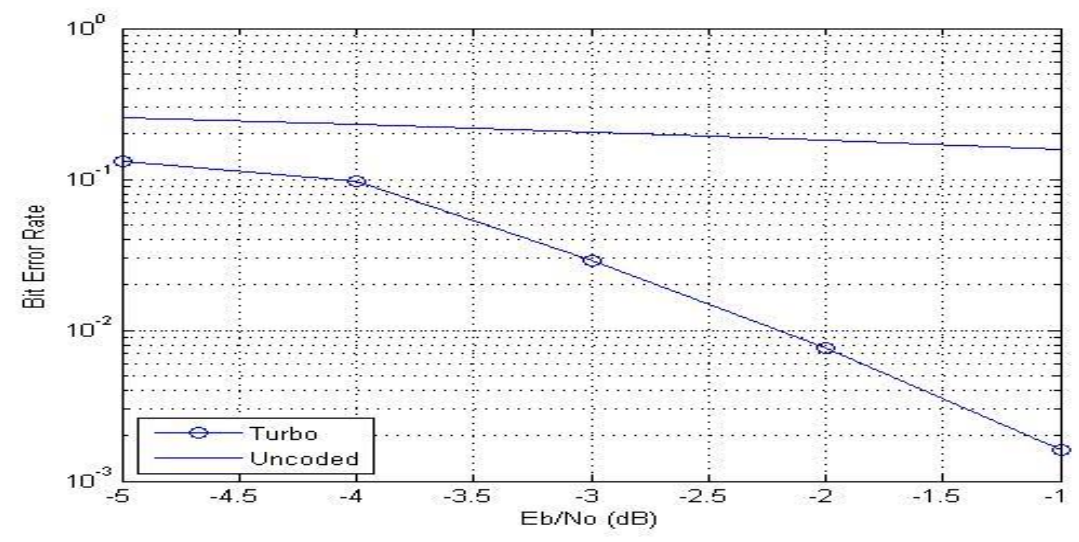

Figure 5. BER with Frame Size 560 Bits

From the figures below, it is obvious that there is a big difference between the BER in coded data and the encoded data via AWGN channel. Through the various values of frame size, the data of the BER are always in between $10^{\wedge}-1$ and $10^{\wedge} 0\left(10^{0}<\mathrm{BER}<10^{-1}\right)$, while in turbo encoder, it can observe that the BER is inversely proportional to the size of the frame. In another word, to achieve high performance and low bit error rate, it is recommended to use a frame with large size, because it can provide more flexibility to the interleaving when it shuffled the data, and it will reduce the ratio of correlation between the neighbor bits.

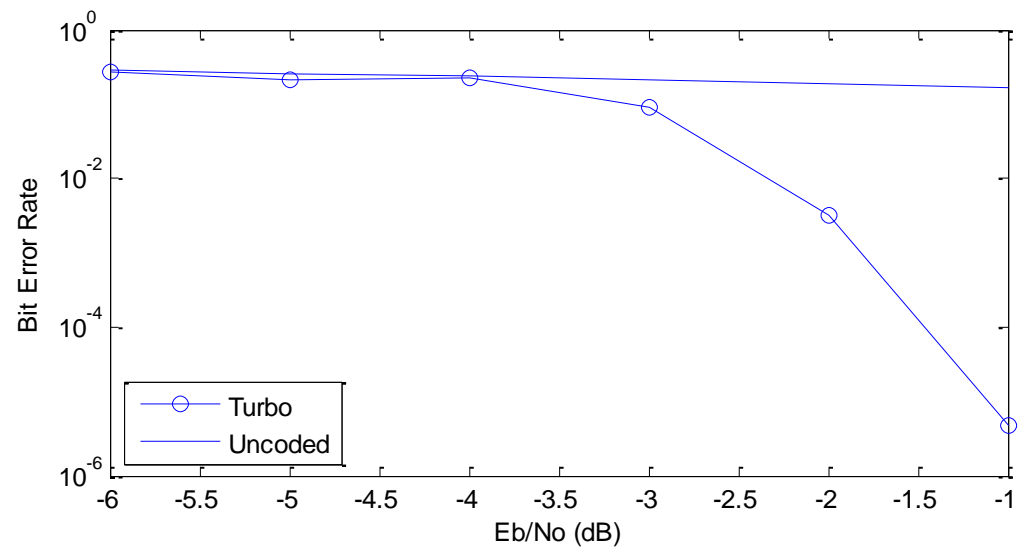

Figure 6. BER with Frame Size 450 Bits

The number of iterations and saturation status: From the graphs, it showed that the increase in numbers of iteration has a positive effect on the performance of turbo code from the point view of BER. However, the more you set a large number of iterations the more you can achieve low bit error rate. Hence, the performance of turbo code will increase. This is because of the exchanging and sharing of the data between the two decoders for many iterations. This will make the decoder give an accurate data but after certain numbers of iteration, the impact becomes insignificant because the decoder has attained a status of saturation. On the contrary, this large number of iteration can cause a remarkable delay and it can verify through the simulation. The graphs and tables below show the bit error in the number of iteration of 2,4 , and 6 . 


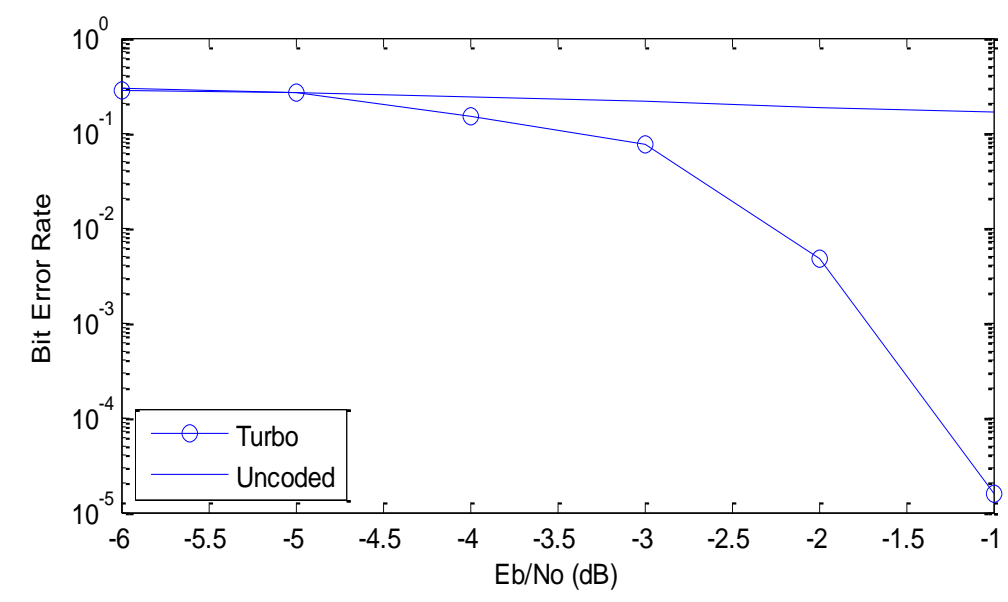

Figure 7. BER with Frame Size $=\mathbf{3 8 0}$ bit with Number off Iteration $=2$

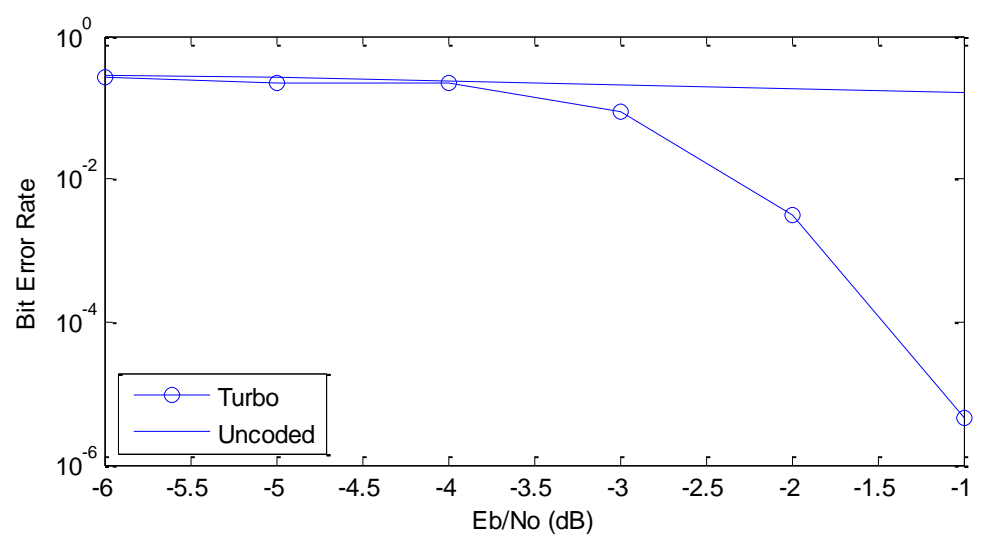

Figure 8. BER with Frame Size $=380$ bit with Number off Iteration=4

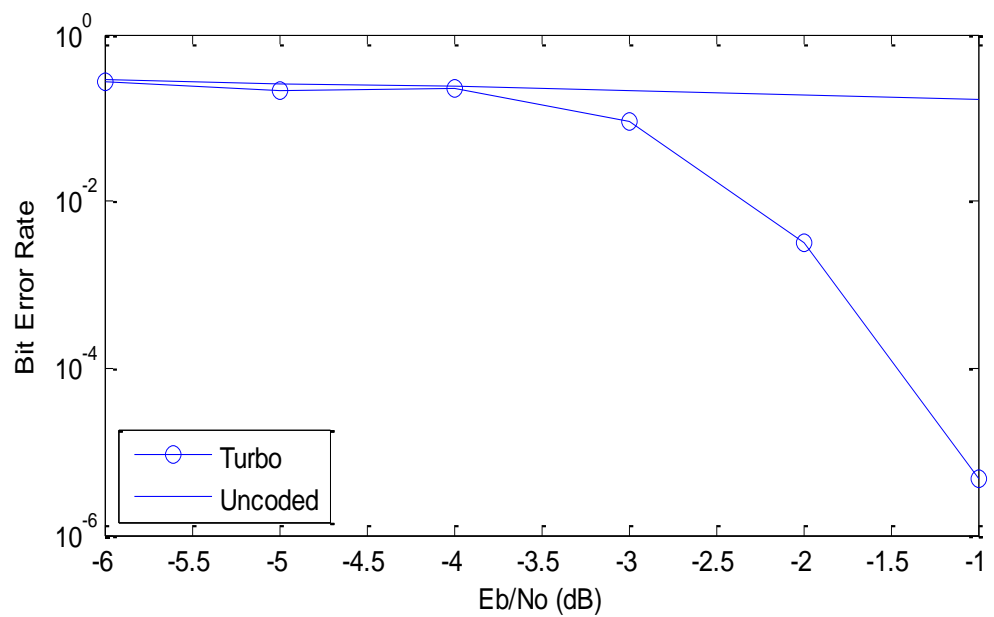

Figure 9. BER with Frame Size $=380$ bit with Number off Iteration $=6$ 


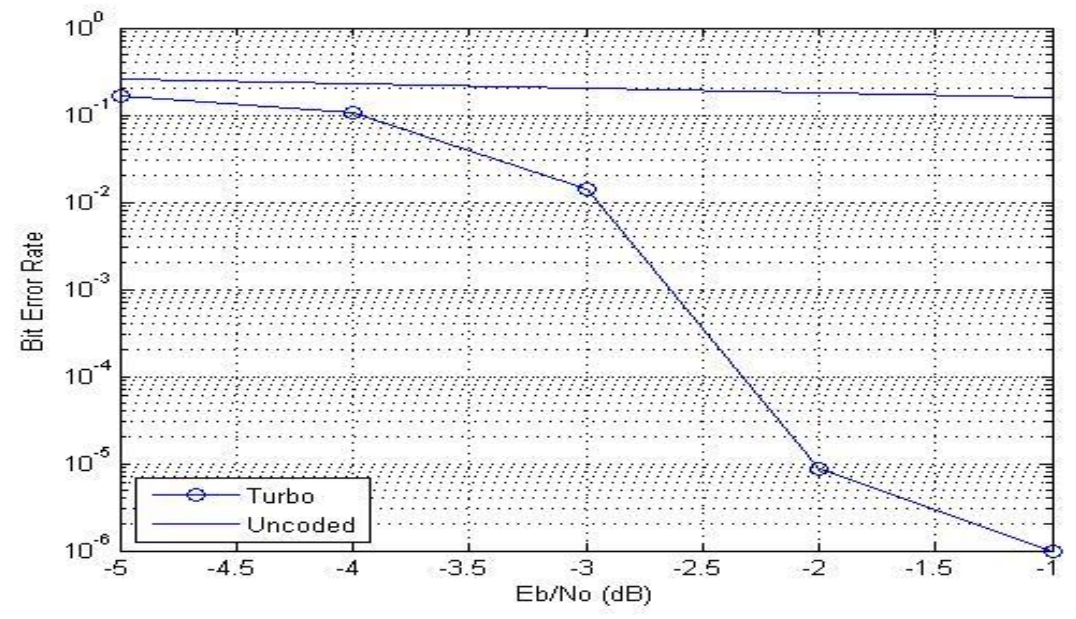

Figure 10. BER with Frame Size $=568$ bit with Number off Iteration $=6$

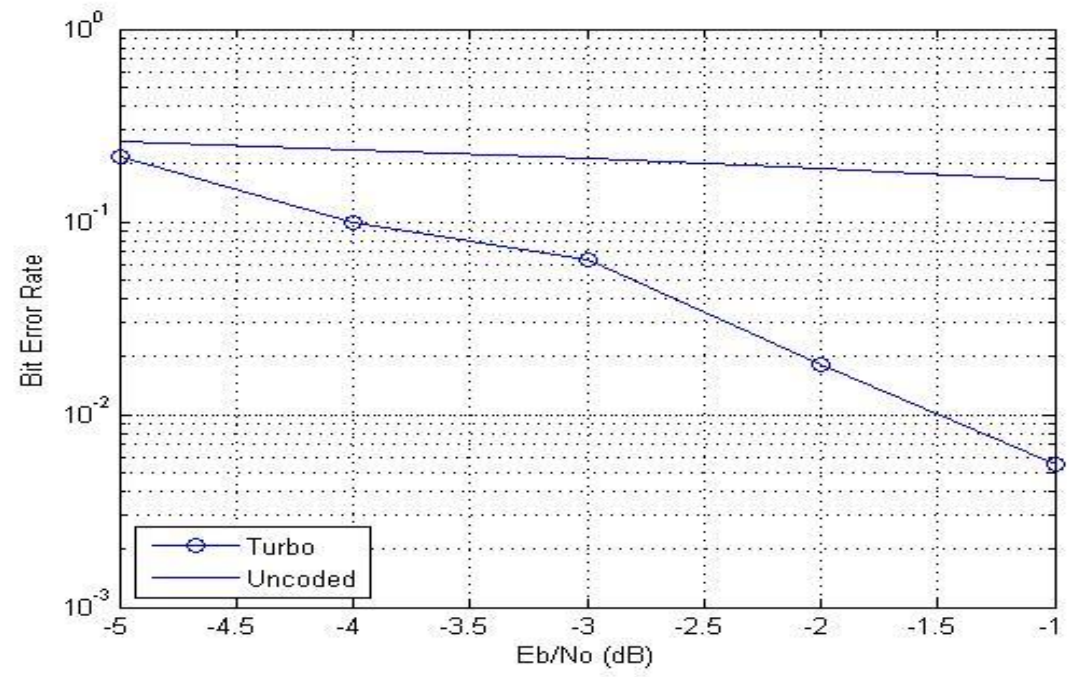

Figure 11. BER with Frame Size $=568$ bit with Number off Iteration $=4$

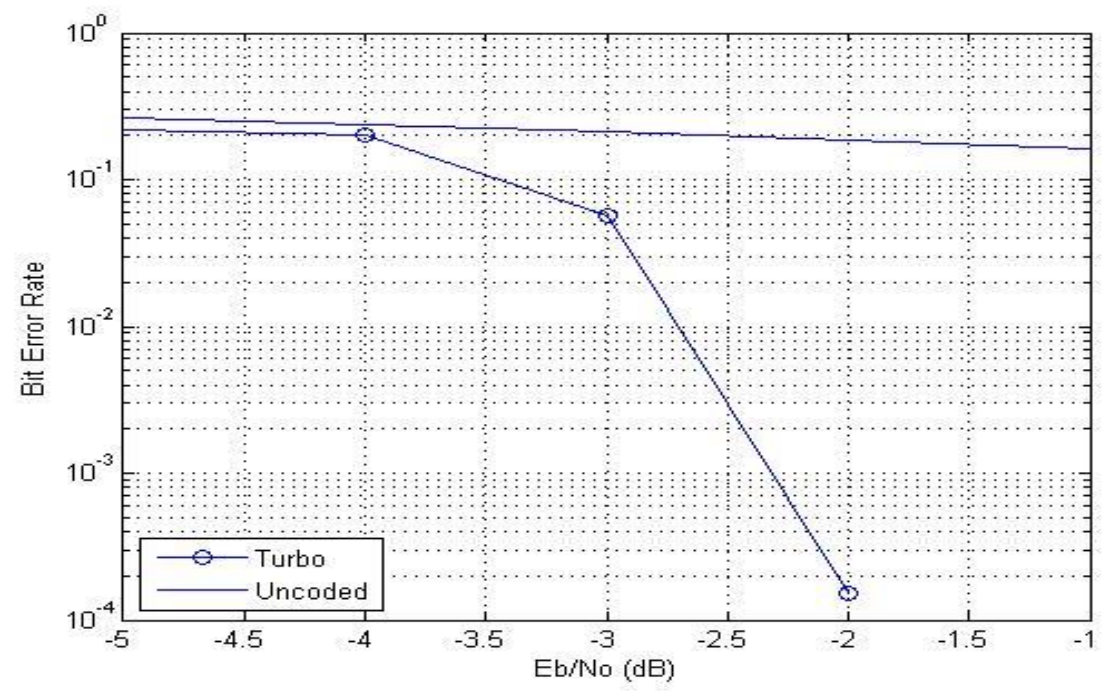

Figure 12. BER with Frame size $=568$ bit with number off Iteration $=2$ 
Table 2. Simulation Result of BER with Number of Iteration $=2$

\begin{tabular}{|l|l|l|l|l|l|l|}
\hline Frame (bit) & Eb/No=-1 & Eb/No=-2 & Eb/No=-3 & Eb/No=-4 & Eb/No=-5 & Eb/No=-6 \\
\hline 380 & 0.2684 & 0.2303 & 0.1566 & 0.0849 & 0.0219 & $3.2369^{*} 10^{\wedge}-4$ \\
\hline 568 & 0.2779 & 0.2414 & 0.2002 & 0.0687 & $4.999^{*} 10^{\wedge}-7$ & 0 \\
\hline 760 & 0.2789 & 0.2171 & 0.1697 & 0.1178 & 0.0138 & $1.3068^{*} 10^{\wedge}-4$ \\
\hline 1144 & 0.2649 & 0.2448 & 0.2080 & 0.1154 & 0.0148 & $9.3889^{*} 10^{\wedge}-5$ \\
\hline 4600 & 0.2649 & 0.2448 & 0.2063 & 0.1291 & 0.0122 & $3.1537^{*} 10^{\wedge}-5$ \\
\hline 6140 & 0.2649 & 0.2448 & 0.2080 & 0.1154 & 0.0148 & $9.3489^{*} 10^{\wedge}-5$ \\
\hline
\end{tabular}

Table2 simulation result of BER with number of iteration $=6$

\begin{tabular}{|l|l|l|l|l|l|l|}
\hline Frame (bit) & Eb/No=-1 & Eb/No=-2 & Eb/No=-3 & Eb/No=-4 & Eb/No=-5 & Eb/No=-6 \\
\hline 380 & 0.2649 & 0.2448 & 0.2080 & 0.1154 & 0.0148 & $0.014899^{*} 10^{\wedge}-5$ \\
\hline 568 & 0.2870 & 0.2518 & 0.1299 & 0.1004 & $5.9471^{*} 10-4$ & $6.6999^{*} 10^{\wedge}-6$ \\
\hline 760 & 0.2553 & 0.2566 & 0.1618 & 0.0987 & $1.3009^{*} 10^{\wedge}-4$ & $6.0000^{*} 10^{\wedge}-7$ \\
\hline 1144 & 0.2640 & 0.2404 & 0.1914 & 0.0734 & $2.7938^{*} 10^{\wedge}-5$ & $5.6995^{*} 10^{\wedge}-6$ \\
\hline 4600 & 0.2673 & 0.2528 & 0.1804 & 0.0993 & $1.9999^{*} 10^{\wedge}-7$ & $1.9999^{*} 10^{\wedge}-7$ \\
\hline 6140 & 0.736 & 0.2283 & 0.1904 & 0.0593 & $3.9992^{*} 10^{\wedge}-7$ & $1.0999^{*} 10^{\wedge}-7$ \\
\hline
\end{tabular}

\section{Comparison}

To enhance this paper, a comparison was made with a related review [10]. However, in this paper, the graph shows different BER with different frames from that of [10] as can be presented in the figure below. As it can be seen, the resultant BER was in the range between 100 and $10-2(100<$ BER<10-2). However, the result in [10] also it supports our result although our simulation achieved a performance higher than $10^{-2}$. 


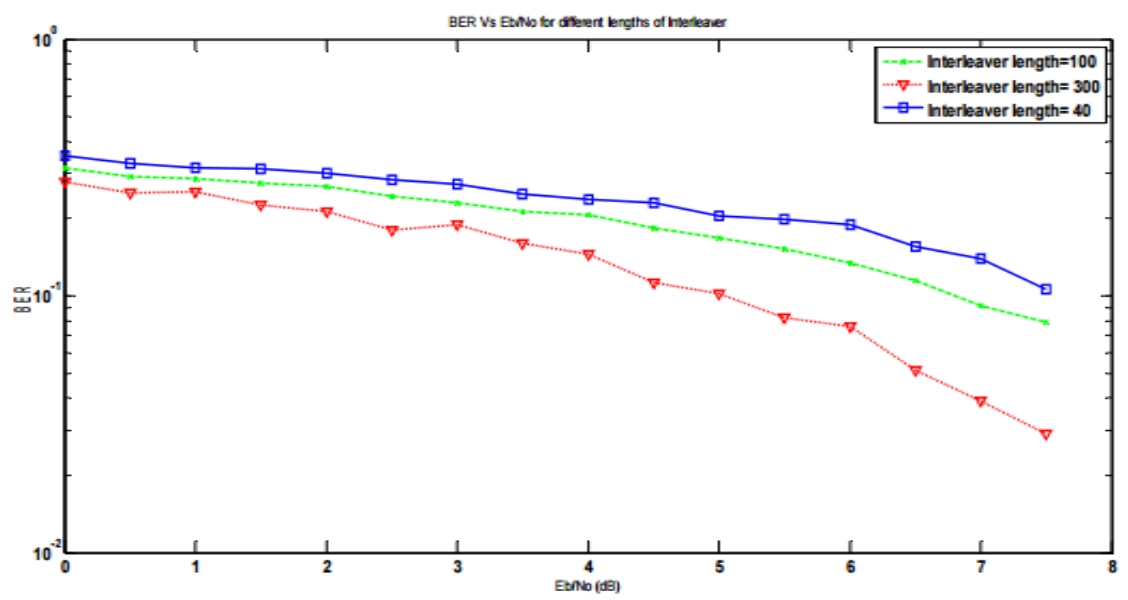

Figure 13. BER with Frame Size $(40,100,300)$ from [10]

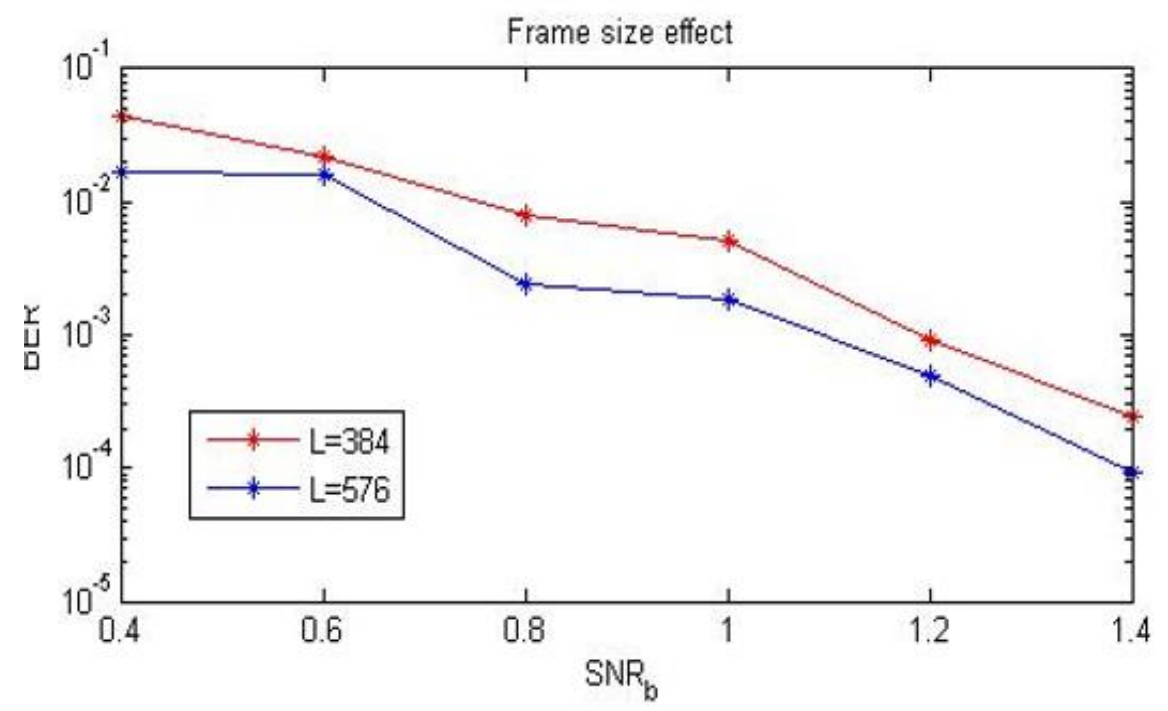

Figure 14. Effect of High Frame in BER[10]

Table 3 Comparison with Previous Work

\begin{tabular}{|l|l|l|}
\hline Frame length & Our research & {$[\mathbf{1 0}]$} \\
\hline 40 & $\mathrm{BER}<10^{-2}$ & $10^{0}<\mathrm{BER}<10^{-1}$ \\
\hline 100 & $\mathrm{BER}<10^{-4}$ & $10^{-0}<\mathrm{BER}<10^{-1}$ \\
\hline 300 & $\mathrm{BER}<10^{-5}$ & $10^{-1}<\mathrm{BER}<10^{-2}$ \\
\hline
\end{tabular}

\section{Conclusion}

In this paper, the performance of turbo code dually highlighted. The turbo code performance identified by processed information, which is encoder and decoder with varying frame lengths and several sequences of iteration. The range of SNR (-1 to -6$) d B$, its proved that the performance of turbo code is very high with the large length of frames. In addition, the BER increased dramatically with the rise in the number of iterations in the 
decoders. A long Time delay in the simulation process is remarkable in both scenarios with a high number of frames and iterations.

\section{References}

[1] O. O. Khalifa, " Applied information theory and coding", IIUM Press Series of Textbook : Science, Technology \& Medicine. IIUM Press, Kuala Lumpur, (2016).

[2] N. Arshad and A. Basit, "Implementation and Analysis of Turbo Codes Using MATLAB", Journal of Expert Systems (JES), vol. 2, no. 1 (2013).

[3] K. Rasadurai and N. Kumaratharan, "Performance enhancement of MC-CDMA system through turbo multi- user detection", Computer Communication and Informatics (ICCCI), 2012 International Conference on, (2012), pp. 1-7.

[4] J. Singh, "Techniques for reduction of multiple access interference in fiber-optic CDMA systems", Journal of Engineering Research and Studies, (2011), p. 7916.

[5] A. Taiwo, S. Seyedzadeh, S. Taiwo, R. K. Z. Sahbudin, M. H. Yaacob, and M. Mokhtar, "Performance and comparison of fiber vibration sensing using SAC-OCDMA with direct decoding techniques", OptikInternational Journal for Light and Electron Optics, vol. 125, no. 17, (2014), pp. 4803-4806.

[6] C. M. Negi, A. Pandey, G. G. Soni, S. K. Gupta, and J. Kumar. "Optical CDMA networks using different detection techniques and coding schemes." International Journal of Future Generation Communication and Networking, vol. 4, no. 3 (2011), pp. 25-34.

[7] M. Hélard, R. L. Gouable, J.-F. Hélard, and J.-Y. Baudais, "Multicarrier CDMA techniques for future wideband wireless networks", Annales des télécommunications, vol. 56, no. 5-6, (2001), pp. 260-274.

[8] R. Joon and A. Nehra, "Performance Evaluation of Symmetric Turbo Codes Using Different Decoding Algorithms", Imperial Journal of Interdisciplinary Research, vol. 2, no. 8, (2016).

[9] V. Garg, "Wireless communications \& networking", Morgan Kaufmann, (2010).

[10] V. Garg, "K. IS-95 CDMA and CDMA2000: Cellular/PCS systems implementation", Pearson Education, (1999).

[11] M. Hélard, R. L. Gouable, J.-F. Hélard and J.-Y. Baudais, "Multicarrier CDMA techniques for future wideband wireless networks", Annales des télécommunications, vol. 56, no. 5-6, (2001), pp. 260-274.

[12] T. S. Rappaport, "Wireless communications: principles and practice, vol. 2", New Jersey: Prentice Hall PTR, (1996).

[13] J. Liu, "Performance analysis of systematic linear codes over AWGN channels", "RFID Technology and Applications (RFID-TA)", 2016 IEEE International Conference on, (2016), pp. 133-137.

[14] M. Yadav and P. Banerjee, "Bit error rate analysis of various interleavers for IDMA scheme", 2016 3rd International Conference on Signal Processing and Integrated Networks (SPIN), (2016). 